\title{
EL CONFINAMIENTO POR EL COVID-19 CAUSA SOLEDAD EN LAS PERSONAS MAYORES. REVISIÓN SISTEMÁTICA
}

\author{
Alba Vázquez Blanco \\ Complejo Asistencial de Zamora \\ alba3108@gmail.com \\ María Baz Codesal \\ Complejo Asistencial de Zamora \\ María Paz Blanco Martín \\ Complejo Asistencial de Zamora
}

Recepción Artículo: 4 diciembre 2020

Admisión Evaluación: 4 diciembre 2020

Informe Evaluador 1: 7 diciembre 2020

Informe Evaluador 2: 9 diciembre 2020

Aprobación Publicación: 10 diciembre 2020

\begin{abstract}
RESUMEN
Antecedentes: La actual pandemia COVID-19 se ha identificado como un posible desencadenante del aumento del sentimiento de soledad y el aislamiento social entre las personas mayores debido a la limitación de movilidad, como la limitación del contacto con sus seres queridos, amigos y actividades de ocio y tiempo libre. En Zamora en Enero del 2020, el 38.41\% de la población eran personas mayores de 60 años. Objetivo: Evaluar el sentimiento de soledad causado por las medidas decretadas para frenar la propagación de la pandemia COVID-19 en las personas mayores de 60 años. Material y método: Se trata de una Revisión Sistemática. Se consultaron las bases de datos: PubMed, Cuiden y Cochrane. Se seleccionaron 13 estudios que relacionaran el confinamiento domiciliario por la Covid-19 con la soledad y los cambios psicológicos que produjo en los ancianos.

Resultados: Las medidas de cuarentena junto con el aislamiento han reducido el contacto social y han aumentado la soledad de las personas mayores, también la ansiedad generalizada y la depresión mayor. Se debe ayudar a adultos mayores a mantener contacto con sus familiares y amigos, para mejorar su salud mental durante el aislamiento. La mortalidad es un factor de miedo básico que rodea la COVID-19, especialmente en la población de edad avanzada. La cuarentena por COVID-19, tiene el potencial de aislar aún más a las personas, lo que resulta en efectos psicológicos negativos que incluyen síntomas de estrés postraumático, confusión e ira. Conclusión: Se evidencia que la soledad se asocia fuertemente con un empeoramiento simultáneo de la depresión, la ansiedad, las preocupaciones sobre el coronavirus y su salud general.
\end{abstract}




\section{EL CONFINAMIENTO POR EL COVID-19 CAUSA SOLEDAD EN LAS PERSONAS MAYORES. REVISIÓN SISTEMÁTICA}

Palabras clave: aislamiento social; social isolation; soledad; Ioneliness; persona mayor; aged adult; Covid-19.

\section{ABSTRACT}

Covid19 confinement causes loneliness in older people. Systematic review. Background: The current COVID-19 pandemic has been identified as a possible trigger for the increase of the feeling of loneliness and the social isolation among the elderly people due to mobility restrictions that has resulted in a limitation of contact with their loved ones and friends and a reduction of their leisure and recreation activities. In the city of Zamora, in January $2020,38.41 \%$ of the population were people over the age of 60 . Objective: To value the feeling of loneliness arising from the measures implemented against the spread of the COVID-19 pandemic in people over the age of 60 . Material and method: This is a Systematic Review. The databases consulted have been the following ones: PubMed, Cuiden and Cochrane. Thirteen (13) studies showing a link between the domiciliary confinement measures implemented against the spread ofCovid-19 home and the feeling of loneliness and with the psychological disorders experienced by the elderly people have been selected. Results: Enacted quarantine measures along with isolation sense have lead into a reduction of the social contact and have increased the feeling of loneliness of the elderly people, as well as a generalized sense of anxiety and major depression. Older adults should be helped to maintain the contact with their families and friends, in order to improve their mental health during isolation. Mortality is a basic fear factor surrounding COVID-19, especially in the elderly population. Quarantine measures arising from the enacted measures against the spread of the Covid-19can potentially create a situation of isolation of people, resulting in negative psychological effects that include symptoms of post-traumatic stress, confusion and anger. Conclusion: It is clear that Ioneliness is strongly associated with simultaneous worsening of depression, anxiety, the concerns about coronavirus and their general health.

Keywords: social isolation; social isolation; Ioneliness; Ioneliness; older person; aged adult; Covid-19.

\section{INTRODUCCIÓN}

En Diciembre de 2019, se informó el primer caso confirmado de infección por SARS-CoV-2 en Wuhan, China, lo que provocó un brote que fue declarado pandemia por la Organización Mundial de la Salud (OMS). Durante los primeros meses de 2020, los gobiernos de muchos países europeos iniciaron medidas de contención con diferentes enfoques que van desde restringir el movimiento de la población y aumentar el distanciamiento social, hasta implementar medidas de aislamiento y cuarentena forzadas. Actualmente nos encontramos en la segunda oleada del brote por el coronavirus 2019 (COVID-19), enfermedad que ha causado miles de muertes y ha dado lugar a medidas de cuarentena impuestas por el gobierno.

La pandemia podría afectar potencialmente los años de vida ajustados a la calidad, lo que podría empeorar aún más el impacto social, clínico y económico de las enfermedades no trasmisibles.

La actual pandemia COVID-19 se ha identificado como un posible desencadenante del aumento del sentimiento de soledad y el aislamiento social entre las personas mayores debido a las restricciones de movimiento, limitando el contacto con sus seres queridos, amigos y actividades de ocio y tiempo libre.

Según el censo del Instituto Nacional de Estadística a 1 de enero de 2020, la población total de la provincia de Zamora era de 171.630 habitantes, el 38.41\% (65.923) eran mayores de 60 años, grupo poblacional con multimorbilidad, definida como múltiples enfermedades no transmisibles concurrentes, como son la hipertensión, dislipemia, obesidad y diabetes mellitus. 
La situación de cuarentena se asocia con importantes consecuencias psicológicas para las personas. El sentimiento de soledad y el aislamiento social se identifican sistemáticamente como factores de riesgo para la mala salud mental y física de las personas mayores. (Chris Noone et al. ,2020)

El grupo de las personas mayores pueden haber experimentado durante la pandemia COVID-19 sentimientos nuevos o empeorados de soledad debido a una interrupción de las actividades sociales en persona, que a menudo son esenciales debido a las limitaciones preexistentes de las condiciones de enfermedades crónicas, discapacidad visual o auditiva, o discapacidad funcional que puede ser especialmente vulnerable a las consecuencias psicológicas asociadas a una mayor gravedad especialmente de sintomatología y mortalidad asociada a este grupo de edad. Un factor que puede contribuir a un mayor riesgo para el padecimiento de problemas psicológicos, tiene que ver con los estereotipos negativos hacia la vejez y, más concretamente, con lo que se ha denominado autopercepción negativa del envejecimiento. (Losada et al., 2020); (Aswin et al., 2020)

\section{OBJETIVO}

El objetivo principal de esta rápida revisión es evaluar el sentimiento de soledad causado por las medidas decretadas para frenar la propagación de la pandemia COVID-19 en las personas mayores de 60 años.

El objetivo secundario es analizar si existen diferencias en función de la edad en ansiedad, tristeza, soledad y comorbilidad ansioso-depresiva en personas jóvenes, de mediana edad y mayores confinadas debido a la pandemia de COVID-19, y explorar la relación entre la autopercepción negativa del envejecimiento y nivel de síntomas psicológicos en función del grupo de edad.

\section{MATERIAL Y MÉTODO}

La tipología seleccionada para la ejecución de esta comunicación fue una Revisión Sistemática. La búsqueda se llevó a cabo durante el mes de Noviembre de 2020 en las bases de datos PubMed, Cuiden y Cochrane. Para la realización de la búsqueda bibliográfica se utilizaron las siguientes combinaciones de palabras clave, "social isolation", "Ioneliness", "aged adult" y "Covid-19" que estuviesen presentes en títulos de publicaciones, resúmenes, palabras clave y en textos completos.

Se seleccionaron aquellos estudios que relacionaran el confinamiento domiciliario por Covid-19 con la soledad y los cambios psicológicos que produjo en los ancianos.

Se encontraron un total de 45 artículos, uno de ellos estaba duplicado en dos bases de datos, de los cuales se seleccionaron para realizar esta revisión 13 artículos tras leer su resumen escritos en español 0 en inglés, seleccionando artículos gratuitos y de pago gracias a la biblioteca virtual de salud del SACYL y que los estudios tratasen de la repercusión psicoafectiva que ha tenido el confinamiento por Covid-19 en los ancianos. La población a estudiar son las personas mayores de 60 años.

Al ser un tema de actualidad no se acotó en el tiempo ya que todas las publicaciones son del año 2020.

\section{RESULTADOS}

Las medidas de cuarentena junto con el aislamiento han reducido el contacto social y como consecuencia han aumentado la soledad de las personas mayores repercutiendo en aumento de los trastornos de salud mental, como la ansiedad generalizada y la depresión mayor. Se ha demostrado que afectan negativamente a elevados resultados de la salud, incluido el aumento de la utilización de la asistencia sanitaria y la mortalidad, así como la desnutrición y la deficiencia de vitamina D. La nutrición, el ejercicio, el entrenamiento cognitivo y el manejo de los factores de riesgo metabólicos 


\section{EL CONFINAMIENTO POR EL COVID-19 CAUSA SOLEDAD EN LAS PERSONAS MAYORES. REVISIÓN SISTEMÁTICA}

y vasculares son todos importantes para mantener el funcionamiento cognitivo y reducir el riesgo de enfermedades crónicas en las personas mayores. (Palmer et al., 2020).

Actividades como ir a la iglesia, la peluquería, pasear, ir al cine, reunirse para tomar un café 0 hacer ejercicio de intensidad baja con amigos, añaden placer a una rutina semanal para las personas mayores. El impacto de la eliminación de tales actividades a través del aislamiento social puede conducir a sentimientos de negatividad.

La depresión se caracteriza por la pérdida de interés en actividades diarias, letargo, falta de energía, perdida notable de peso o por lo contrario aumento de peso, cambio en el apetito, ansiedad, agitación o inquietud, sus manifestaciones clínicas son dolores corporales inexplicables, dificultad en la concentración y en la toma de decisiones, dormir demasiado o de lo contrario muy poco, cambios en los hábitos alimenticios 0 en el apetito, ira, irritabilidad o mayor frustración. Los signos y síntomas de la ansiedad que puede manifestar una persona mayor son los siguientes: sentirse nervioso, inquieto o tenso, tener la sensación mantenida de peligro, aumento de la frecuencia cardiaca, hiperventilación, aumento de la sudoración, temblor inexplicable, disminución de la concentración, higiene deficiente del sueño, náuseas, vómitos o diarrea, aumento del letargo y luchan para controlar las preocupaciones; las manifestaciones clínicas, son las siguientes: afecta a todos los aspectos biopsicosociales de la vida cotidiana, los individuos son incapaces de controlarla y les causa malestar, puede llegar a causar depresión, abuso de sustancias o exacerbación de otros problemas de salud mental, con comportamientos o pensamientos suicidas. (Baker \& Clark, 2020)

La mortalidad es un factor de miedo básico que rodea a la COVID-19, especialmente en la población de edad avanzada, y se valora que muchos ya están sufriendo signos de ansiedad y depresión en torno a esto. Las noticias en los medios de comunicación pueden causar a angustia y a la vez aumentarla.

Es importante que cuando los adultos mayores están socialmente aislados, se les ayude a mantener contacto con sus familiares y amigos, y es probable que este sea el método más eficaz para mejorar la salud mental de una persona durante el aislamiento COVID-19, sus falta de habilidades de tecnología puede ser notable en las generaciones más mayores, y algunas siguen siendo ingenuas de Internet, especialmente aquellas con un estatus financiero deficiente y un nivel educativo más bajo 0 mismamente la edad. Por ejemplo, (Chris Noone, 2020) afirma: "las videollamadas se ha visto que pueden ayudar a estas personas a mantenerse conectadas durante la crisis actual ampliando el círculo social o aumentando la frecuencia del contacto con los conocidos" (P.1)

Los aspectos beneficiosos que aportan las videollamadas son: tranquilidad, disminución de la soledad, Ilanto en representación de la emoción, humanización, disminuyen la ansiedad, el miedo, la soledad, el enfado y la ira, aumentan la motivación y sobre todo la esperanza de la curación al ver a través de una pantalla a sus seres queridos.

En el estudio de (Beam \& Kim, 2020) menciona a diferentes grupos de personas: adolescentes adultos jóvenes y mayores y. "Aunque la soledad puede tener grandes efectos negativos en la salud física, cognitiva y psicológica de los adultos mayores, la soledad durante la COVID-19 puede tener un efecto aún mayor en las poblaciones de adolescentes y adultos jóvenes. Los miembros de las generaciones más jóvenes reportan sentirse solos con más frecuencia que los adultos mayores. Más del $60 \%$ de los adultos mayores informaron que nunca se sintieron solos, mientras que menos del 10\% informaron una soledad severa. Si bien se presume que la soledad aumenta con la edad, particularmente en la edad adulta mayor, cuando la salud física disminuye y se acumulan pérdidas interpersonales, este no es necesariamente el caso. Más bien, los sentimientos de soledad tienden a estabilizarse 0 incluso a disminuir ligeramente con la edad. A medida que continúa el distanciamiento social debido a la pandemia de la COVID-19, es probable que los adolescentes, los adultos 
jóvenes y los adultos mayores experimenten un empeoramiento de la soledad, especialmente si las personas importantes se pierden por la COVID-19."

En el estudio que realizó (Losada et al., 2020), se demuestra que las personas mayores informan de menores niveles de problemas de salud mental comparadas con jóvenes. Las personas mayores informan de puntuaciones significativamente menores de ansiedad y tristeza que las de mediana edad. En relación con la soledad las personas de mediana edad informan de una soledad significativamente menor que la informada por las personas mayores, quienes a su vez informan de significativamente menos soledad que las personas jóvenes. Con respecto a la ansiedad, hay más personas jóvenes y menos personas mayores de las esperadas con niveles elevados, sin embargo, con niveles bajos de ansiedad hay más personas mayores que jóvenes. Con respecto a la tristeza, hay menos personas mayores de las esperadas con niveles elevados y en niveles bajos se encuentra a menos personas jóvenes y más mayores de las esperadas. Con respecto a la soledad, hay más personas jóvenes de las esperadas con niveles elevados y menos personas jóvenes y más de mediana edad de las esperadas con niveles bajos. Con respecto a los casos con niveles elevados conjuntos de ansiedad y tristeza (comórbidos), hay menos personas mayores y más personas jóvenes de las esperadas. Las personas con sintomatología elevada comórbida en ansiedad y tristeza tienen puntuaciones significativamente superiores en autopercepción negativa que el resto de los grupos. Los resultados de este trabajo podrían estar respaldando una mayor capacidad de afrontamiento adaptativo de las personas mayores ante situaciones de estrés como la vivida ante la COVID-19, a pesar de que informan de peor salud subjetiva. Otro de los resultados centrales de este estudio es la significativa relación entre los auto estereotipos sobre el envejecimiento y el bienestar psicológico a lo largo de todo el ciclo vital. En todos los grupos de edad se han encontrado asociaciones directas entre la autopercepción negativa sobre el propio envejecimiento y la ansiedad, la depresión y la soledad. Los síntomas depresivos y ansiosos se asocia con un curso más prolongado del malestar, así como con importantes efectos perjudiciales sobre la salud física y la discapacidad en personas mayores.

En la investigación de (Tilburg, 2020), compara la soledad antes y durante la pandemia de la COVID-19, los adultos mayores holandeses que vivían en la comunidad en mayo de 2020 mostraron que durante la pandemia de la COVID-19, la soledad social aumentó ligeramente y la soledad emocional aumentó mucho más fuertemente. La comparación con la situación siete meses antes (octubre de 2019) demuestra las consecuencias de la pandemia.

Según el estudio realizado por (Aswin et al., 2020), demuestran en su estudio que las personas que estaban socialmente aisladas eran más propensas a tener dificultad para encontrar ayuda con las necesidades funcionales, particularmente el baño, varios participantes informaron de un uso exitoso de la tecnología para mantener las conexiones con las actividades de la comunidad y sus seres queridos, y que sus relaciones con la tecnología cambiaron durante la pandemia. Sin embargo, varios participantes mencionaron el malestar con la tecnología 0 no tener un acceso adecuado a internet 0 equipos que limitan las interacciones sociales de ayuda. El uso limitado de la tecnología a menudo llevó a la incapacidad de interactuar con una red social más amplia y a que las personas se limitaran a interactuar con los miembros de la familia o con nadie en absoluto. Los participantes describieron además las barreras para obtener ayuda con las tareas domésticas, la cocina y un transporte. En general, el $54 \%$ de los participantes atribuyó un empeoramiento de los sentimientos de soledad a la pandemia de coronavirus al menos una vez durante el período de estudio. En cuanto a la salud psicológica, las personas que reportaban un empeoramiento de la soledad eran más propensas a tener un empeoramiento de la depresión debido a COVID-19. Así como empeoramiento de la ansiedad. Además, los participantes tenían preocupaciones por el empeoramiento de la salud 


\section{EL CONFINAMIENTO POR EL COVID-19 CAUSA SOLEDAD EN LAS PERSONAS MAYORES. REVISIÓN SISTEMÁTICA}

debido a retrasos en la atención médica y la inseguridad alimentaria. Entre estos participantes, varios experimentaron puntuaciones de soledad que aumentaron en gravedad con el tiempo 0 puntuaciones de soledad que se mantuvieron altas con el tiempo. En las respuestas abiertas, estas personas describieron restricciones COVID-19 que amplifican las pérdidas sociales previas (por ejemplo, viudez), dificultad para usar alternativas basadas en la tecnología para socializar, sentimientos abrumadores de estar atrapados y soledad que afecta su salud física y mental. Otros reportaron un empeoramiento de la soledad que fue relativamente leve con el tiempo. Estos participantes alcanzaron una sensación en desarrollo de aburrimiento, pero manejando su soledad a través de mantenerse ocupados con las actividades en el hogar y la adopción de nuevas tecnologías.

Según el estudio realizado por (Li \& Wang, 2020), los términos sociodemográficos muestran que las mujeres y las personas más jóvenes tienen probabilidades significativamente más altas de soledad que los hombres y las personas mayores. También encontramos que las personas que viven con una pareja y tienen un empleo remunerado tienen probabilidades significativamente menores de soledad frecuente que aquellas que no viven con una pareja y están sin trabajo. Además, en comparación con las personas sin síntomas relacionados con COVID-19, las personas que alguna vez tuvieron síntomas, y especialmente las personas que actualmente tienen síntomas, tienen probabilidades significativamente más altas de soledad frecuente.

En esta investigación de (Levi-Belz \& Aisenberg, 2020) los datos indicaron que los ciudadanos mayores de Israel, experimentaron una carga psicológica debido a la crisis provocada por la COVID19. Un $45,5 \%$ de ellos, informaron de una necesidad de asistencia especial y el $30 \%$ informó un aumento de los niveles de angustia junto con una soledad severa.

En el estudio de (Ishikawa, 2020), los adultos mayores se ven afectados de manera desproporcionada. Su menor respuesta inmune y la mayor tasa de comorbilidades médicas se traducen en una mayor proporción de hospitalizaciones y muertes relacionadas con virus en comparación con los adultos más jóvenes. Los adultos mayores están siguiendo la progresión de la pandemia con una variedad de reacciones. Mientras que muchos se han adaptado a las precauciones y las directivas de aislamiento, otros están experimentando un dolor abrumador, así como la sensación de pavor caracterizada como dolor anticipatorio.

Según (Padalaet al., 2020) las cuarentenas de la COVID-19, tienen el potencial de aislar aún más a las personas, lo que resulta en efectos psicológicos negativos que incluyen síntomas de estrés postraumático, confusión e ira. En una muestra comunitaria de adultos jóvenes, más de la mitad de Ios encuestados calificaron el impacto psicológico de la emergencia COVID-19 como "moderado" o "severo". Aquellos que tienen un deterioro cognitivo preexistente y viven solos pueden tener un mayor riesgo de descompensación psicológica debido a la orientación desde el hogar en comparación con aquellos que no tienen deterioro cognitivo y viven con la familia.

Al contrario de todos los resultados anteriores, en el estudio de (García-Fernández et al., 2020) se objetiva, que los mayores de 60 años son menos vulnerables que los participantes más jóvenes a sufrir depresión y estrés agudo, además, no han mostrado diferencias en los niveles de ansiedad durante el pico de la pandemia en comparación con el grupo menor de 60 años, por lo que las personas mayores no pueden considerarse especialmente vulnerables al desarrollo de ansiedad, depresión y estrés agudo durante el pico de la pandemia de COVID-19 en España. En cuanto a las variables ambientales estudiadas, los resultados han mostrado que, a diferencia de lo visto en situaciones de aislamiento y distanciamiento social por diferentes circunstancias, nuestros datos no muestran una relación entre la soledad y el aumento de la ansiedad, depresión y estrés agudo. Además, la soledad como parte del aislamiento impuesto por la cuarentena no se ha asociado con las con- 
secuencias psicológicas negativas que suelen acompañar a la desconexión social en circunstancias distintas a la pandemia actual.

\section{DISCUSIÓN}

Las personas mayores son el grupo más vulnerable ante la enfermedad producida por el SARSCoV-2, presentan peor pronóstico, por síndromes geriátricos, por su morbilidad y la fragilidad asociada al envejecimiento. Las medidas adoptadas por los distintos países para frenar la infección afectan principalmente a los ancianos, las autoridades deben ser un apoyo fundamental tanto para los que viven solos 0 en comunidad y verse protegidos. Ellos han formado, forman y seguirán formando parte de nuestra historia y necesitan ayuda para sobrellevar esta situación. Además de los problemas físicos que acarrea la enfermedad son también igual de importantes los problemas psicológicos, estar lejos de sus seres queridos, especialmente en momentos dolorosos.

\section{CONCLUSIONES}

Es imposible predecir o comprender el impacto que está causando la pandemia de la COVID-19 sobre la soledad en las personas mayores. Lo que sí está claro es que la soledad se asocia fuertemente con un empeoramiento simultáneo de la depresión, la ansiedad, las preocupaciones sobre el coronavirus y las preocupaciones sobre su salud general. A medida que pasa el tiempo, la soledad puede mejorar porque los adultos mayores se adaptan a esta circunstancia 0 mantenerse persistente 0 empeorada con el tiempo. Las interacciones basadas en la tecnología pueden aliviar el aislamiento, como son las videollamadas.

Se puede revelar que una autopercepción negativa del envejecimiento puede favorecer la aparición de problemas psicológicos como la ansiedad, la depresión o la soledad, puesto que pueden predisponer a una menor implicación en comportamientos saludables y a un incremento en la reactividad emocional.

Se puede demostrar, como hemos visto en los resultados anteriores, que las personas jóvenes tienen niveles superiores de ansiedad, tristeza y soledad que las personas mayores asociados al confinamiento por problemas de salud como la COVID-19: a menor edad, mayor riesgo de presentar sintomatología emocional negativa porque supone un mayor cambio en sus rutinas de actividad y un mayor riesgo de pérdidas 0 alteraciones con relación a sus valores o metas, más vinculadas a la actividad social, educativa o económica cotidiana (p. ej., problemas o cambios laborales y económicos asociados a estos, suspensión o retrasos indefinidos de actividades formativas importantes, imposibilidad de actividades sociales, de ocio y diversión, etc.), e incluso a la amenaza de la propia vida. Estas circunstancias podrían respaldar estudios como el de Swift et al. (2014), que encuentran que las condiciones económicas son fundamentales para explicar la relación entre bienestar y edad.

Se requerirán más investigaciones para comprender plenamente el impacto de la pandemia COVID-19 en la soledad y el aislamiento en las poblaciones mayores.

\section{REFERENCIAS BIBLIOGRÁFICAS:}

Noone, C., McSharry, J., Smalle, M., Burns, A., Dwan, K., Devane, D., \& Morrissey, E. C. (2020). Video calls for reducing social isolation and loneliness in older people: a rapid review. The Cochrane database of systematic reviews, 5(5), CD013632. https://doi.org/10.1002/14651858.CD013632.

Palmer, K., Monaco, A., Kivipelto, M., Onder, G., Maggi, S., Michel, JP, Prieto, R., Sykara, G. y Donde, S. (2020). El impacto potencial a largo plazo del brote de COVID-19 en pacientes con enfermedades no transmisibles en Europa: consecuencias para un envejecimiento 


\section{EL CONFINAMIENTO POR EL COVID-19 CAUSA SOLEDAD EN LAS PERSONAS MAYORES. REVISIÓN SISTEMÁTICA}

saludable. Investigación clínica y experimental sobre el envejecimiento , 32 (7), 11891194. https://doi.org/10.1007/s40520-020-01601-4

Beam, C. R., \& Kim, A. J. (2020). Psychological sequelae of social isolation and loneliness might be a larger problem in young adults than older adults. Psychological trauma : theory, research, practice and policy, 12(S1), S58-S60. https://doi.org/10.1037/tra0000774

Van Tilburg, TG, Steinmetz, S., Stolte, E., van der Roest, H. y de Vries, DH (2020). Soledad y salud mental durante la pandemia de COVID-19: un estudio entre adultos mayores holandeses. Las revistas de gerontología. Serie B, Ciencias psicológicas y ciencias sociales , gbaa111. Publicación anticipada en línea. https://doi.org/10.1093/geronb/gbaa111

Kotwal, AA, Holt-Lunstad, J., Newmark, RL, Cenzer, I., Smith, AK, Covinsky, KE, Escueta, DP, Lee, JM y Perissinotto, CM (2020). Aislamiento social y soledad entre los adultos mayores del área de la bahía de San Francisco durante las órdenes de refugio en el lugar COVID-19. Revista de la Sociedad Americana de Geriatría , 10.1111 / jgs.16865. Publicación anticipada en línea. https://doi.org/10.1111/jgs.16865

Li, LZ y Wang, S. (2020). Prevalencia y predictores de trastornos psiquiátricos generales y soledad durante COVID-19 en el Reino Unido. Investigación en psiquiatría , 291 , 113267. https://doi.org/10.1016/j.psychres.2020.113267

Levi-Belz, Y., \& Aisenberg, D. (2020). Together we stand: Suicide risk and suicide prevention among Israeli older adults during and after the COVID-19 world crisis. Psychological trauma : theory, research, practice and policy, 12(S1), S123-S125. https://doi.org/10.1037/tra0000667

García-Fernández, L., Romero-Ferreiro, V., López-Roldán, PD, Padilla, S., y Rodriguez-Jimenez, R. (2020). Salud mental en ancianos españoles en época de brote de COVID-19. Revista estadounidense de psiquiatría geriátrica: publicación oficial de la Asociación Estadounidense de Psiquiatría Geriátrica , 28 (10), 1040-1045. https://doi.org/10.1016/j.jagp.2020.06.027

Ishikawa R. Z. (2020). I may never see the ocean again: Loss and grief among older adults during the COVID-19 pandemic. Psychological trauma : theory, research, practice and policy, 12(S1), S85-S86. https://doi.org/10.1037/tra0000695

Swift, H. J., Vauclair, C. M., Abrams, D., Bratt, C., Marques, S., \& Lima, M. L. (2014). Revisiting the paradox of well-being: the importance of national context. The journals of gerontology. Series B, Psychological sciences and social sciences, 69(6), 920-929. https://doi.org/10.1093/geronb/gbu011

Padala, KP, Parkes, CM y Padala, PR (2020). Impacto neuropsicológico y funcional de COVID-19 en el deterioro cognitivo leve. American Journal of Alzheimer's Disease \& Other Dementiasr . https://doi.org/10.1177/1533317520960875

Whitehead, BR y Torossian, E. (2020). La experiencia de los adultos mayores de la pandemia de COVID-19: un análisis de métodos mixtos de estrés y alegría. El gerontólogo, gnaa126. Publicación anticipada en línea. https://doi.org/10.1093/geront/gnaa126 